\title{
Smooth Transportation of Liquid Metal Droplets in a Microchannel as Detected by a Serially Arranged Capacitive Device
}

Satoshi Konishi ( $\square$ konishi@se.ritsumei.ac.jp)

Department of Mechanical Engineering, College of Science and Engineering, Ritsumeikan University, Kusatsu, 525-8577, Japan

Yuugo Kakehi

Ritsumeikan University

Fuminari Mori

Ritsumeikan University

Shinji Bono

Ritsumeikan University

\section{Research Article}

Keywords: Gallium alloy liquid metals (Ga-LMs), electric conductivity, patterned electrodes

Posted Date: January 16th, 2021

DOl: https://doi.org/10.21203/rs.3.rs-144799/v1

License: (a) (i) This work is licensed under a Creative Commons Attribution 4.0 International License. Read Full License

Version of Record: A version of this preprint was published at Scientific Reports on March 29th, 2021. See the published version at https://doi.org/10.1038/s41598-021-86394-w. 


\title{
Smooth transportation of liquid metal droplets in a microchannel as detected
}

\section{by a serially arranged capacitive device}

\author{
Satoshi Konishi ${ }^{1,2,3^{*}}$, Yuugo Kakehi ${ }^{2}$, Fuminari Mori ${ }^{2}$, Shinji Bono ${ }^{1,2,3}$ \\ ${ }^{1}$ Department of Mechanical Engineering, College of Science and Engineering, Ritsumeikan University, \\ Kusatsu, 525-8577, Japan \\ ${ }^{2}$ Graduate Course of Science and Engineering, Ritsumeikan University, Kusatsu, 525-8577, Japan \\ ${ }^{3}$ Ritsumeikan Global Innovation Research Organization, Ritsumeikan University, Kusatsu, 525-8577, \\ Japan \\ *konishi@se.ritsumei.ac.jp
}

\begin{abstract}
Gallium alloy liquid metals (Ga-LMs) possessing fluidity, electric conductivity, and low toxicity are attractive for use in flexible devices and microfluidic devices. However, the oxide skin of Ga-LMs in the atmosphere adheres to the microchannel surface, preventing the transportation of GaLMs in the channel. We introduced liquid with Ga-LMs into a channel with a radius of $500 \mu \mathrm{m}$ to prevent the oxide skin of the Ga-LM from adhering to the channel. Then, we found that the cylindrical shape of the channel enabled smooth transportation of Ga-LMs independently of both the liquid and the channel material. The liquid introduced with Ga-LMs not only prevents adhesion but also improves the spatial controllability of Ga-LMs in the channel. We can control the position of Ga-LMs with 100 $\mu \mathrm{m}$ resolution using highly viscous $(>10 \mathrm{cSt})$ liquid. In addition, we combined the microchannel with patterned electrodes, fabricating a serially arranged capacitive device. The local capacitance detected by the patterned electrodes changed by more than $6 \%$ via the smooth transportation of Ga-LMs. The analysis results based on an equivalent circuit quantitatively agree with our experimental results. We can modulate the serially arranged capacitors using the smooth transportation of Ga-LMs in the channel.
\end{abstract}

\section{Introduction}

Liquid metal (LM) has both fluidity and high electrical conductivity and is an appropriate material for electrical wiring and sensors in flexible devices ${ }^{1-3}$. Since LM is soft and has a smooth shape, it is easily introduced into microchannels, indicating that it has promising microfluidic device 
applications, such as in switches and valves ${ }^{4}$. A well-known LM is mercury, which shows fluidity and high electric conductivity at room temperature. Hence, mercury has been used broadly in electric switches, diodes, and pressure gauges. However, because of the large burden on the environment and the high toxicity of mercury, its usage has recently been avoided ${ }^{5,6}$. Gallium alloy-LM (Ga-LM) is a fascinating alternative to mercury because Ga-LM shows low toxicity as well as fluidity and electrical conductivity 7,8 .

The application of Ga-LM to microdevices presents the following challenges: in the atmosphere, gallium at the surface is oxidized, and as a result, a thin (1-3 nm) oxide skin is spontaneously formed ${ }^{2,9}$. The oxide skin is adhesive to surfaces, which inhibits Ga-LM from being transported smoothly in the microchannel and causes disconnection and deterioration of flexible devices ${ }^{10}$. In addition, the gallium oxide skin is an electric insulator $(\sim \text { a few } M \Omega)^{11}$. Thus, the application of Ga-LM based on its electrical conductivity is difficult without nontrivial treatment to remove the oxide skin.

By chemical surface treatment with a strong acid or base, the oxide skin can be removed, and thus both the fluidity and the electric conductivity can be maintained ${ }^{12,13}$. However, Ga-LM in the atmosphere continues to be oxidized spontaneously. To take advantage of the promising properties of Ga-LM with chemical treatment, continuous chemical treatment is necessary; that is, microdevices must contain a strong acid or base with Ga-LM. Therefore, the chemical treatment requirement is a disadvantage in terms of practical device applications.

Recently, it was reported that liquid, such as water, introduced with Ga-LM into microchannels can prevent the oxide skin from adhering to the surfaces of microfluidic devices ${ }^{14}$. The fluidity of Ga-LM dispersed in liquid can be maintained without harsh chemicals, which is an advantage over chemical treatment. However, the mechanism for preventing gallium oxide from adhering is unclear. In principle, with this method, it is impossible to remove the oxide skin, making applications based on electric resistance difficult without additional treatment. Therefore, it is important to use electrical properties that can be detected without direct $\operatorname{contact}^{15,16}$.

In this paper, we investigated the mechanism of the smooth transportation of Ga-LM in microchannels, focusing on the geometry of and materials in the microfluidic devices. After introducing Ga-LM into the microchannel, we applied pressure to the Ga-LM. To control the position of the Ga-LM in the microchannel, we prevented Ga-LMs from adhering to the surface of the channel and stably transported Ga-LMs while keeping its shape intact. In particular, we quantitatively estimated the reproductivity, which is an important parameter for the spatial control of Ga-LMs. Then, we fabricated a serially arranged capacitive device in which Ga-LMs are transported smoothly. We modulated the local capacitance using the smooth transportation of Ga-LMs.

\section{Results}


Figure 1 (a) shows a microscopic image of a Ga-LM and water in the cylindrical microchannel (diameter: $\phi=600 \mu \mathrm{m}$ ). The Ga-LM did not adhere to the surface of the channel and maintained a smooth, intact shape. While pressure was applied to the inlet, the moving Ga-LMs never adhered to the channel surface. Then, to investigate the effect of the channel material of the microfluidic device, we introduced Ga-LMs into cylindrical channels composed of Teflon ( $\phi=1 \mathrm{~mm}$ and $0.8 \mathrm{~mm}$ ), vinyl chloride $(\phi=1 \mathrm{~mm})$, and glass $(\phi=0.6 \mathrm{~mm})$. As a result, we confirmed that the Ga-LM is transported smoothly without adhesion to the surface of the channels. In addition, we observed the transportation of Ga-LMs with silicone oil, Fluorinert, or vegetable oil, whose chemical properties differ from each other. As a result, we confirmed that the oxide skin of Ga-LM does not adhere to the channel surface independently of the liquid used. These results suggest that the materials that make up the channel and the liquid do not affect the transportation of Ga-LMs.

Figure 1 (b) shows a microscope image of the Ga-LM and water in a square pole-shaped channel. Since the Ga-LM adhered to the channel surface just its injection with water, the Ga-LM does not show fluidity in the square pole-shaped channel. Next, we fabricated a hybrid microchannel composed of glass and polydimethylsiloxane (PDMS), which is a typical combination in fluid microelectromechanical systems (MEMSs) ${ }^{17,18}$. However, the Ga-LM in the square pole-shaped hybrid microchannel adhered to the channel surface. Therefore, the cylindrical shape of the microchannel is important for the smooth transportation of Ga-LMs.

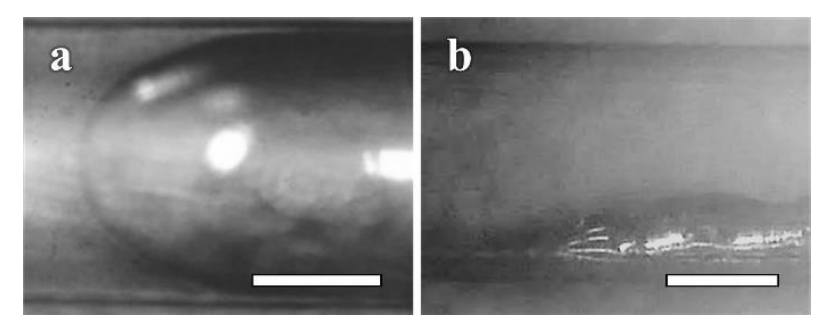

Figure 1. Microscope images of the Ga-LM and water (a) in the cylindrical microchannel and (b) in the square pole-shaped channel. The scale bars indicate $250 \mu \mathrm{m}$.

Since the spatial resolution of the Ga-LM in the microchannel is directly related to the detection resolution of devices, we focused on the spatial control of Ga-LMs. We sealed the outlet and repeatedly applied positive $(50 \mathrm{kPa})$ and negative pressure on Ga-LMs in the microchannel. After 50 compression processes, we measured the spatial deviation from the initial position. As a result, the deviation of Ga-LMs in water was larger than $2 \mathrm{~mm}$. This is caused by the time lag between the switching of the pressure direction and that of the transporting direction of Ga-LMs due to the inertia of Ga-LMs in low viscosity liquid. Then, we used highly viscous liquids, that is, vegetable or silicone oil, instead of water. Figure 2 (a1-a3) shows optical microscopy images of Ga-LMs and silicone oil under positive pressure. Although the kinetic viscosity of silicone oil is much higher than that of water, 
the Ga-LM is transported smoothly. In contrast, the Ga-LM is transported in an inverse direction under negative pressure, as shown in Fig. 2 (b1-b3). We summarized the liquids used and the kinetic viscosity and deviation of Ga-LMs from the initial position in Table 1. When Ga-LMs disperse in a highly viscous liquid, the deviation becomes smaller than the observation resolution of the microscope $(<100$ $\mu \mathrm{m})$.
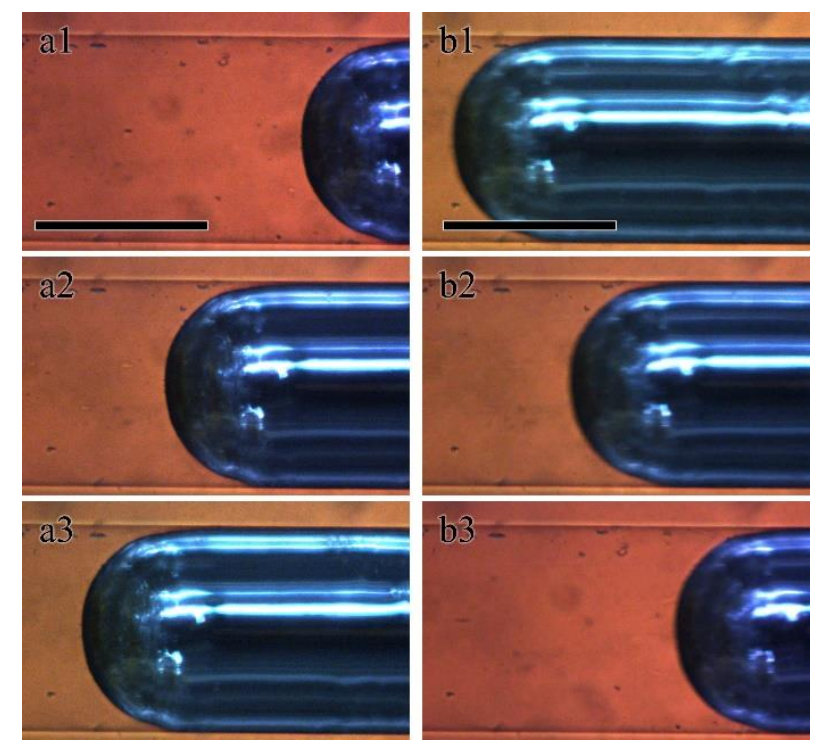

Figure 2. (a1-3) Optical microscopy images of Ga-LMs in silicone oil under positive pressure. The snapshots were taken every $5 \mathrm{~s}$. (b1-b3) Optical microscopy images of Ga-LMs in silicone oil under negative pressure. Snapshots were taken every $1 \mathrm{~s}$. The scale bars indicate $500 \mu \mathrm{m}$.

Table 1 Kinetic viscosity and the deviation from the initial position after 50 compression processes.

\begin{tabular}{ccc} 
Material & Kinetic viscosity $\left(25^{\circ} \mathrm{C}\right)[\mathrm{cSt}]$ & Deviation $[\mathrm{mm}]$ \\
\hline Water & 0.893 & $>2$ \\
Vegetable oil & 10 & $<0.1$ \\
Silicon oil & 100 & $<0.1$
\end{tabular}

To fabricate a serially arranged capacitive device using the smooth transportation of Ga-LM droplets, we investigated the relation between capacitance and the position of Ga-LMs in a microchannel. Then, we sandwiched the microchannel between patterned electrodes and investigated the local capacitance of Ga-LMs introduced in the channel. Figure 3 (a) shows a schematic cross section of the serially arranged capacitive device. The diameter of the channel was $1.0 \mathrm{~mm}$. We defined the thickness of the channel composed of PDMS as $d$ and used a channel with $d=2.3 \mathrm{~mm}$. The GaLM in the microchannel is enlarged in Fig. 3 (b). We connected the syringe pump to the inlet to control 
the position of Ga-LMs by pressure. Figure 3 (c) shows a photograph of the serially arranged capacitive device. The microchannel was sandwiched between two glass substrates, on which we patterned three pairs of aluminum electrodes. Via capacitance measurements, a pair of electrodes is connected to an inductance-capacitance-resistance (LCR) meter. We measured $C_{0}$ and $C(x)$, where $C_{0}$ and $C(x)$ are the capacitance without Ga-LMs, i.e., the channel is occupied by pure silicone oil, and the capacitance as a function of the position of Ga-LMs, $x$ (we took the origin at the center of our device), respectively. $C_{0}$ was found to be $3-4 \mathrm{pF}$. Since the dielectric constant of silicone oil is almost the same as that of PDMS, and $C_{0}=\varepsilon S / d$, substituting the experimental values of $\varepsilon \sim 3 \times 10^{-11} \mathrm{~F} / \mathrm{m}, S \sim$ $6 \times 10^{-5} \mathrm{~m}^{2}$, and $d \sim 2 \times 10^{-3} \mathrm{~m}$, we obtain $C_{0} \sim 1 \mathrm{pF}$, which agrees with the experimental value.

The red, green, and blue markers in Fig. 3 (c) indicate the capacitance measured at the left, center, and right electrodes, respectively. Here, we normalized $C(x)$ as $C(x) / C_{0}$ to neglect the difference between the shapes of those electrodes. When Ga-LMs reach a position between the electrodes, the normalized capacitance $C(x) / C_{0}$ takes the maximum value. In contrast, when the Ga-LMs are far from the electrodes, $C(x) / C_{0}$ takes a constant minimum value. The change in $C(x) / C_{0}$ is larger than $2 \%$, which indicates that we can modulate the serially arranged capacitors using the smooth transportation of Ga-LMs in the microchannel.

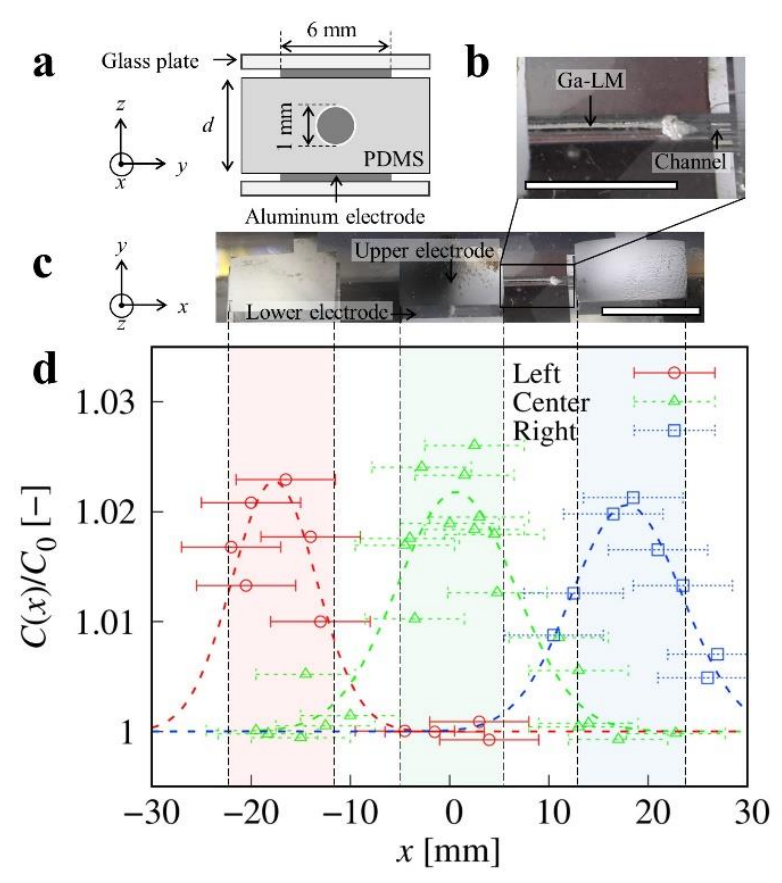

Figure 3. (a) Schematic cross section perpendicular to the microchannel. (b) An enlarged photograph of Ga-LMs in the microfluidic channel. The diameter of the channel is $1.0 \mathrm{~mm}$. The scale bar indicates $5 \mathrm{~mm}$. (c) A perspective photograph of the serially arranged capacitive device. The channel was sandwiched by upper and lower aluminum electrodes at three points, that is, at the left, center, and right. The scale bar indicates $10 \mathrm{~mm}$. (d) Normalized capacitance $C(x) / C_{0}$ as a function of the position 
of Ga-LMs, $x$. Red, green, and blue markers indicate the capacitance measured at the left, center, and right electrodes, respectively. We provided a visual guide to indicate the position of the electrodes.

One of the important factors that determined the change in the capacitance is the volume ratio of Ga-LMs to PDMS. We fabricated microfluidic devices composed of PDMS with a thickness of 1.4$5.5 \mathrm{~mm}$. The thickness of PDMS must be greater than $1 \mathrm{~mm}$ because the diameter of the channel is 1 $\mathrm{mm}$. Then, we sandwiched the microfluidic device between uniform electrode substrates and measured $C_{0}$ and $C$, where $C$ is the capacitance under the condition that the channel is occupied by Ga-LMs. Figure 4 shows the PDMS thickness dependence of the normalized capacitance $C / C_{0}$. Red markers indicate the $C / C_{0}$ measured by uniform electrodes. The blue markers indicate the maximum change in $C(x) / C_{0}$ estimated from the results shown in Fig. 3 (c). When the thickness of a device is $1.4 \mathrm{~mm}$, the $C / C_{0}$ is larger than $6 \%$. In addition, $C / C_{0}$ monotonically decreases with increasing thickness.

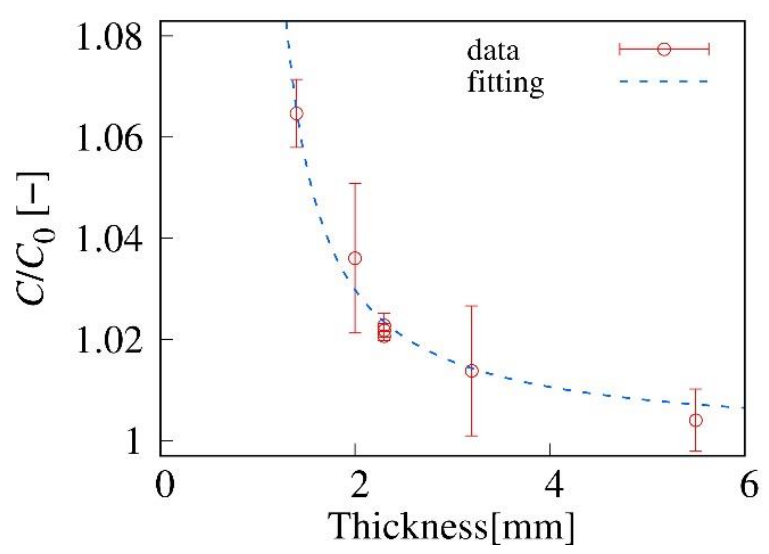

Figure 4. Dependence of the capacitance of Ga-LMs on the PDMS thickness. Red and blue markers indicate the experimental results measured by uniform electrodes and the maximum change estimated from the results shown in Fig. 3 (c), respectively. The diameter of the channel is $1 \mathrm{~mm}$. The green line is the best fit curve obtained using equation (5.a).

\section{Discussion}

\section{Smooth transportation of Ga-LMs}

In the cylindrical channel, the Ga-LM is subjected to a uniform force from the channel. As a result, the liquid layer is uniformly inserted between Ga-LMs and the channel, preventing Ga-LMs from adhering to the channel. In contrast, Ga-LMs in the square pole-shaped channel are under heterogeneous forces from the channel. For example, the force from the flat part of the channel is different from that of the edge. Since Ga-LMs are forced against the surface of the channel by heterogeneous forces, the oxide skin adheres to the channel. Therefore, to smoothly transport Ga-LMs 
through the microchannel, the shape of the cross section must be a circle, independent of the liquid and channel material.

\section{Change in the capacitance with and without Ga-LMs}

Since silicone oil in the channel is replaced by Ga-LMs via smooth transportation, the local capacitance increases. To quantitatively analyze the change in the capacitance, we constructed an equivalent circuit of our device with two approximations: (i) the distribution of electric charge in the parallel plane to the electrodes is uniform, and (ii) the capacitance out of the device is negligible because the capacitances of PDMS and the channel are larger than that of air.

Figure 5 shows a schematic cross section of the channel in PDMS with thickness $d$ and a width of $6 \mathrm{~mm}$. We superimposed the equivalent circuit on the cross section, as shown in Fig. 5. The $z$-axis is perpendicular to the electrodes. We regarded PDMS in the ranges of $(d+1) / 2<z<d$ and $0<$ $\mathrm{z}<(d-1) / 2$ as capacitors with capacitance,

$C_{\mathrm{p} 1}=C_{\mathrm{p} 3}=\varepsilon_{\mathrm{p}} S \frac{2}{d-1}$

where $\varepsilon_{\mathrm{p}}$ and $S$ are the dielectric constant of PDMS and the electrode area, respectively. We regarded the channel and PDMS in the range of $(d-1) / 2<z<(d+1) / 2$ as two parallel capacitors because we neglected the heterogeneity of electric charges in the perpendicular plane to the $z$-axis. The capacitance of PDMS in the range of $(d-1) / 2<z<(d+1) / 2$ is given as

$C_{\mathrm{p} 2}=\varepsilon_{\mathrm{p}}\left(1-\frac{\lambda}{6}\right) S$

where $\lambda$ is the geometric parameter that corresponds to the area ratio of the channel to PDMS. For geometrical reasons, $\lambda<1$. Since silicone oil is mainly composed of PDMS, we regarded the dielectric constant of silicone oil as $\varepsilon_{\mathrm{p}}$. Then, the capacitance of the channel occupied by silicone oil, $C_{\mathrm{SO}}$, and that occupied by Ga-LMs, $C_{\mathrm{LM}}$, are denoted as

$$
C_{\mathrm{f}}=\left\{\begin{array}{lr}
C_{\mathrm{SO}}=\varepsilon_{\mathrm{p}} \frac{\lambda}{6} S & \text { (silicon oil) } \\
C_{\mathrm{LM}}=\alpha C_{\mathrm{SO}} & (\text { Ga-LM) }
\end{array}\right.
$$

where $\alpha$ is the ratio of the capacitance with Ga-LMs to that with silicone oil. Equations (1-3) indicate that the total capacitance of the equivalent circuit is given as

$\frac{1}{C}=\frac{1}{C_{\mathrm{p} 1}}+\frac{1}{C_{\mathrm{f}}+C_{\mathrm{p} 2}}+\frac{1}{C_{\mathrm{p} 3}}$

The normalized capacitance $C / C_{0}$ is given as

$\frac{C}{C_{0}}=\frac{d}{d-\delta(\alpha, \lambda)}$

$\delta(\alpha, \lambda)=\frac{\lambda(\alpha-1)}{6+\lambda(\alpha-1)}$ 
Equation (5.a) indicates that the electrode gap $d$ is modified as $d-\delta$ by replacing silicone oil with Ga-LMs in the channel. We fitted the experimental results with equation (5.a), where $\delta$ is the only fitting parameter, and showed the best fit curve in Fig. 4 as the green line. Our model based on the equivalent circuit agrees approximately with the experimental results. A slight quantitative disagreement is thought to be caused by our approximations, where we neglected the electric heterogeneity in the parallel plane to the electrodes. The fitting result indicates that $\delta \sim 70 \mu \mathrm{m}$, and $\alpha$ $=1.5-6$. This result suggests that the capacitance increases 1.5-6 times by introducing Ga-LMs into the channel.

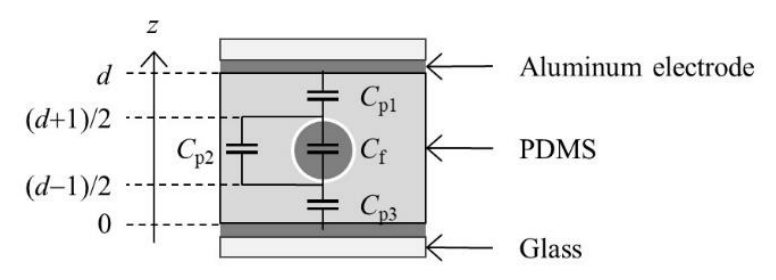

Figure 5. Schematic cross section of the channel and the equivalent circuit.

\section{Motion and position detection of transported Ga-LM droplets in a microchannel}

We succeeded in changing the capacitance of serially arranged capacitors using the smooth transportation of Ga-LMs. In the serially arranged capacitive device, the normalized capacitance $C(x) / C_{0}$ depends on the relative positions of Ga-LMs and the electrodes. When the Ga-LM is far from the electrodes, $C(x) / C_{0}$ takes a constant minimum value. After intruding into the channel sandwiched by the electrodes, $C(x) / C_{0}$ starts increasing. $C(x) / C_{0}$ eventually reaches a maximum value at the position where the center of the Ga-LM corresponds with that of the electrodes. These results indicate that $C(x) / C_{0}$ monotonously depends on the volume ratio of Ga-LMs in the channel between the electrodes.

For microfabrication, (i) the length of the Ga-LM and the width of the electrodes should correspond with each other to improve the spatial resolution of the Ga-LM near the center of the electrodes. Under the condition that the length of Ga-LMs is not equal to the width of electrodes, the spatial deviation of Ga-LMs from the center of the electrodes is not accompanied by a change in $C(x) / C_{0}$ because the volume fraction of Ga-LMs is invariant near the center of the electrodes. (ii) The length of Ga-LMs should be longer than the interval between pairs of electrodes. Unless a part of GaLMs overlaps with electrodes, the spatial change in Ga-LMs does not cause a change in $C(x) / C_{0}$. Considering the fact that Ga-LMs must be larger than the diameter of the channel, we found that the limitation of the microfabrication is $\sim 1$ pattern $/ \mathrm{mm}$. The average transportation velocity is $\sim 10 \mathrm{~mm} / \mathrm{s}$. Therefore, the signal frequency was estimated as $\sim 10 \mathrm{~Hz}$.

We modulated serially arranged capacitors using the smooth transportation of Ga-LM droplets, 
which conversely indicates that we can sense the position of Ga-LMs in the channel from the change in capacitance. The change in the capacitance is $10^{-2} \mathrm{pF} / \mathrm{mm}$. Taking into account the resolution of the LCR meter $\left(10^{-2} \sim 10^{-3} \mathrm{pF}\right)$, the position of Ga-LMs can be detected with a $100 \mu \mathrm{m}$ resolution. The deviation after the transport process was repeated was found to be approximately $100 \mu \mathrm{m}$. Thus, we can sense the position of Ga-LMs based on serially arranged capacitors with a comparable resolution with spatial controllability.

\section{Conclusion}

In this paper, we smoothly transported Ga-LMs through a microchannel and modulated the serially arranged capacitors. We showed that (1) Ga-LMs introduced into a cylindrical channel with liquid are transported smoothly independently of the liquid and the channel material because the GaLM in the cylindrical channel is subjected to a uniform force from the channel, (2) the position of GaLMs can be controlled with a resolution of $100 \mu \mathrm{m}$ using highly viscous ( $10 \mathrm{cSt}$ ) liquid, and (3) the local capacitance of the patterned electrode device can be modulated by the position of Ga-LMs in the channel. These results suggest that the smooth transportation of Ga-LMs through a channel is applicable for the modulation of serially arranged capacitors. Moreover, we showed the possibility of applying a serially arranged capacitive device as a spatial sensor of Ga-LMs even in a microfabrication system.

\section{Method}

\section{Observation of Ga-LMs in a microchannel}

We introduced Ga-LMs (eutectic gallium indium stannum, Zairyo-ya.com) and liquid into a microchannel composed of PDMS (DuPont Toray Specialty Materials K.K.). We used water, vegetable oil (Kadoya Sesame Mill inc.), Fluorinert (3M), and silicone oil (MOMENTIVE) as the liquids. One side of the through-hole of the PDMS microchannel was an inlet, and the other side was an outlet. We fabricated two kinds of channels, that is, a cylindrical channel and a square pole-shaped channel. The diameter of the cylindrical channel $\phi$ was $600 \mu \mathrm{m}-1 \mathrm{~mm}$, and the dimensions of the square pole channel were $500 \mu \mathrm{m} \times 500 \mu \mathrm{m}$. For observation of Ga-LMs in the microchannel, we used a microscope (DIGITAL MICROSCOPE VHX-500F, KEYENCE) and a transmission optical microscope (MX9430, MEJI TECHNO).

\section{Position control of Ga-LMs in microchannel}

We introduced Ga-LMs and liquid from the inlet. Then, we sealed the outlet with epoxy glue, where a small amount of air between the liquid and outlet was maintained. To transport Ga-LMs from the inlet to the outlet, we applied $30 \mathrm{kPa}$ pressure to the inlet for $5 \mathrm{~s}$. For transportation in the backward direction, we removed the pressure for $5 \mathrm{~s}$. 


\section{Position of Ga-LMs and serially arranged capacitors}

A PDMS microchannel with $\phi=1 \mathrm{~mm}$ and diameter $d$ was sandwiched by glass plates whose surface was patterned with aluminum electrodes. Electrodes were connected with an LCR meter (LCR METER, KEYSIGHT). We injected Ga-LMs and silicone oil into the microchannel with a syringe pump. To control the position of Ga-LMs in the microchannel, we applied pressure to the channel. For capacitance measurements, aluminum electrodes connected to an LCR meter were applied to an AC electric field at $2 \mathrm{~V}$ and $500 \mathrm{kHz}$. We measured the capacitance and the position of Ga-LMs simultaneously.

The total capacitance of PDMS and the microchannel is approximately $\varepsilon S / d$, where $\varepsilon, S$, and $d$ are the dielectric constant of PDMS, the electrode area, and the electrode gap, respectively. The resolution of capacitance increases with decreasing electrode gap. The minimum electrode gap is the diameter of the microchannel. For this reason, we designed an electrode gap on a similar order to the diameter of the microchannel $(\sim 1 \mathrm{~mm})$.

We used two kinds of electrode substrates to evaluate the capacitance.

(1) Patterned electrodes (Fig. 6 (a1-2))

We patterned the upper glass substrate with three aluminum electrodes whose dimensions were $6 \mathrm{~mm} \times 10 \mathrm{~mm}$. The intervals of electrodes were $16 \mathrm{~mm}$. The lower glass substrate was patterned with three aluminum electrodes whose width was $10 \mathrm{~mm}$ with a $16 \mathrm{~mm}$ spacing. We connected a pair of electrodes to an LCR meter to measure the local capacitance. Based on the balance between spatial and measurement resolutions, the electrode area $S$ was set to $60 \mathrm{~mm}^{2}$.

(2) Uniform electrodes (Fig. 6 (b1-2))

We deposited uniform aluminum electrodes on glass substrates to accurately measure the capacitance. PDMS microchannels with a width of $6 \mathrm{~mm}$ and diameter $d$ were sandwiched by uniform electrode substrates whose electrode area $S$ was $\sim 400 \mathrm{~mm}^{2}$. Upper and lower electrodes were connected to an LCR meter to measure the total capacitance of the fluidic device.

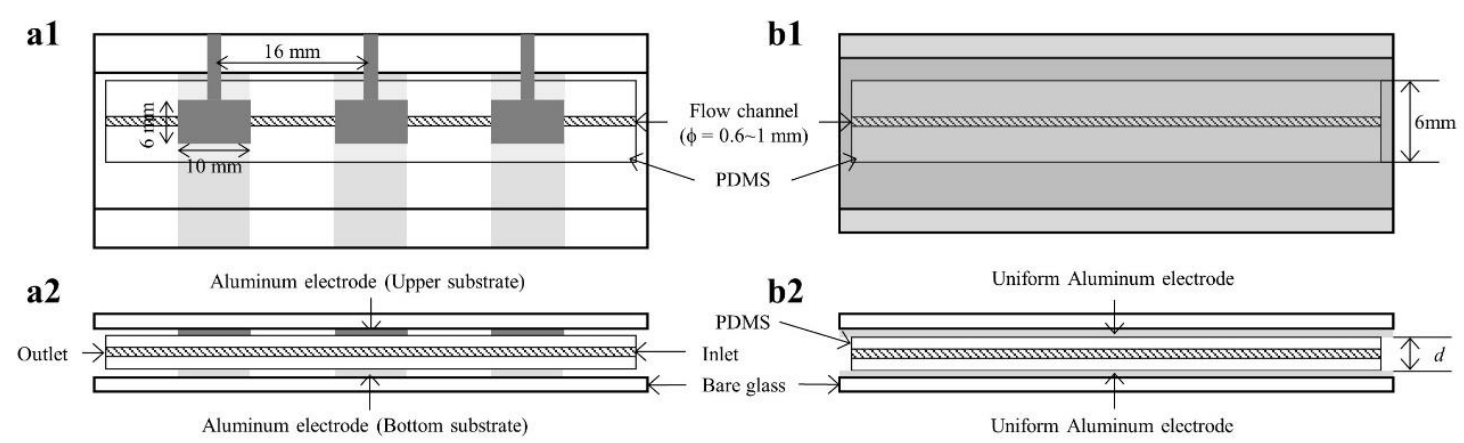


Figure 6. (a1) Schematic top view and (a2) schematic side view of the patterned electrode. We deposited three aluminum electrodes with dimensions of $6 \mathrm{~mm} \times 10 \mathrm{~mm}$ on the upper glass substrate with intervals of $16 \mathrm{~mm}$. We deposited three aluminum electrodes whose width was $10 \mathrm{~mm}$ on the lower glass substrate with an interval of $16 \mathrm{~mm}$. (b1) Schematic top view and (b2) schematic side view of uniform electrodes.

\section{Data availability}

All data are included in this published article.

\section{References}

1. Otake, S., Mori, F. \& Konishi, S. Common Flexible Channel Structure for Integration of ThreeAxis Force Sensor and Soft Micro Actuator. in 2019 IEEE 32nd International Conference on Micro Electro Mechanical Systems (MEMS) 652-655 (2019). doi:10.1109/MEMSYS.2019.8870621.

2. Zheng, Y., He, Z., Gao, Y. \& Liu, J. Direct Desktop Printed-Circuits-on-Paper Flexible Electronics. Sci. Rep. 3, 1786 (2013).

3. Kazem, N., Hellebrekers, T. \& Majidi, C. Soft Multifunctional Composites and Emulsions with Liquid Metals. Adv. Mater. 29, 1605985 (2017).

4. Tang, S.-Y., Lin, Y., Joshipura, I. D., Khoshmanesh, K. \& Dickey, M. D. Steering liquid metal flow in microchannels using low voltages. Lab. Chip 15, 3905-3911 (2015).

5. Boening, D. W. Ecological effects, transport, and fate of mercury: a general review. Chemosphere 40, 1335-1351 (2000).

6. Clarkson, T. W., Magos, L. \& Myers, G. J. The toxicology of mercury--current exposures and clinical manifestations. N. Engl. J. Med. 349, 1731-1737 (2003). 
7. Liu, T., Sen, P. \& Kim, C. Characterization of Nontoxic Liquid-Metal Alloy Galinstan for Applications in Microdevices. J. Microelectromechanical Syst. 21, 443-450 (2012).

8. Dickey, M. D. Stretchable and Soft Electronics using Liquid Metals. Adv. Mater. 29, 1606425 (2017).

9. Dickey, M. D. Emerging Applications of Liquid Metals Featuring Surface Oxides. ACS Appl. Mater. Interfaces 6, 18369-18379 (2014).

10.Boley, J. W., White, E. L., Chiu, G. T.-C. \& Kramer, R. K. Direct Writing of Gallium-Indium Alloy for Stretchable Electronics. Adv. Funct. Mater. 24, 3501-3507 (2014).

11.Mori, F., Hirata, A., Kakehi, Y. \& Konishi, S. An Effect of a Needle Electrode for the Ohmic Contact Between an Electrode and Liquid Metal Droplet in Electrical Switching. in 2020 IEEE 33rd International Conference on Micro Electro Mechanical Systems (MEMS) 957-960 (2020). doi:10.1109/MEMS46641.2020.9056437.

12.Khoshmanesh, K. et al. Liquid metal enabled microfluidics. Lab. Chip 17, 974-993 (2017).

13.Kim, D. et al. Recovery of Nonwetting Characteristics by Surface Modification of Gallium-Based Liquid Metal Droplets Using Hydrochloric Acid Vapor. ACS Appl. Mater. Interfaces 5, 179-185 (2013).

14.Khan, M. R., Trlica, C., So, J.-H., Valeri, M. \& Dickey, M. D. Influence of Water on the Interfacial Behavior of Gallium Liquid Metal Alloys. ACS Appl. Mater. Interfaces 6, 22467-22473 (2014). 
15.So, J.-H. et al. Reversibly Deformable and Mechanically Tunable Fluidic Antennas. Adv. Funct.

Mater. 19, 3632-3637 (2009).

16.Chen, C., Whalen, J. \& Peroulis, D. Non-Toxic Liquid-Metal 2-100 GHz MEMS Switch. in 2007

IEEE/MTT-S International Microwave Symposium 363-366 (2007).

doi:10.1109/MWSYM.2007.380446.

17.Fujii, T. PDMS-based microfluidic devices for biomedical applications. Microelectron. Eng. 61-

62, 907-914 (2002).

18.Zhou, J., Ellis, A. V. \& Voelcker, N. H. Recent developments in PDMS surface modification for microfluidic devices. ELECTROPHORESIS 31, 2-16 (2010).

\section{Acknowledgements}

This work was partially supported by Ritsumeikan Global Innovation Research Organization.

\section{Author contribution}

S.K. conceived the direction of research and experiment. S.K., Y.K., F.M., and S.B. performed experiments. S.K. and S.B. wrote the manuscript. All author discussed the results and reviewed the manuscript.

\section{Competing interests}

The authors declare no competing interest. 
Figures
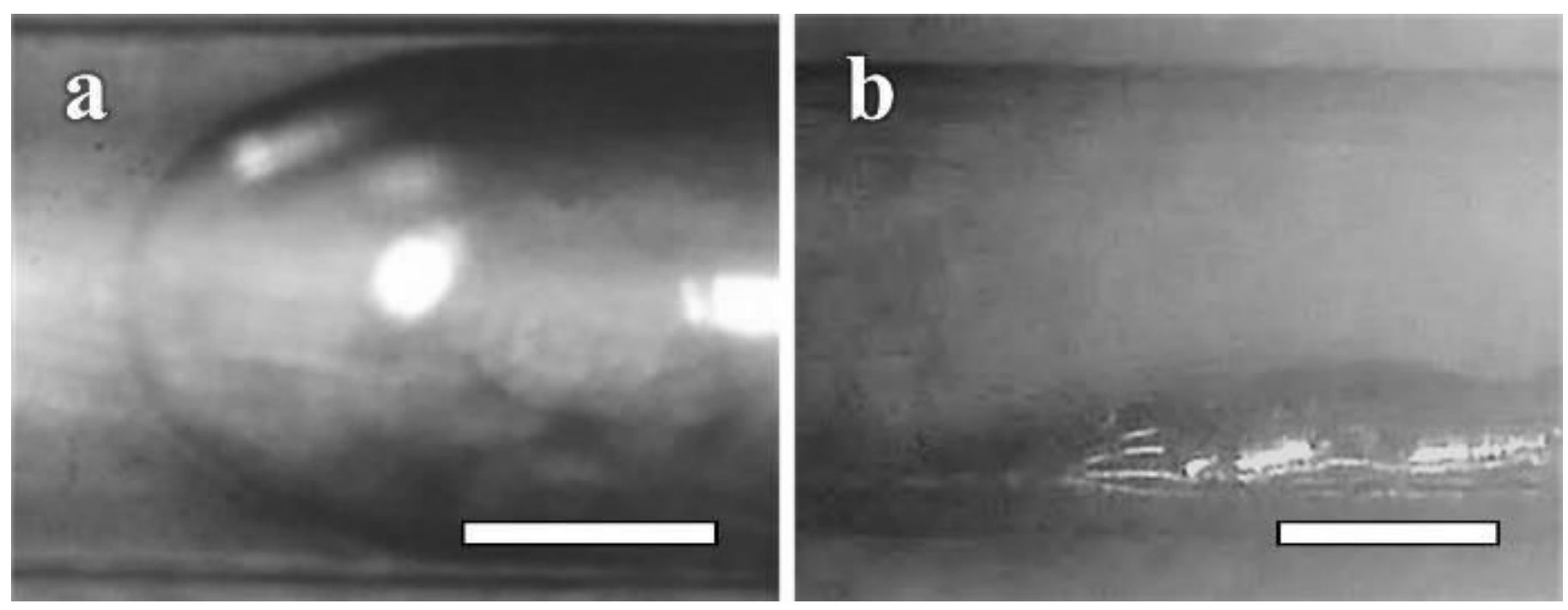

\section{Figure 1}

Microscope images of the Ga-LM and water (a) in the cylindrical microchannel and (b) in the square poleshaped channel. The scale bars indicate $250 \mu \mathrm{m}$. 

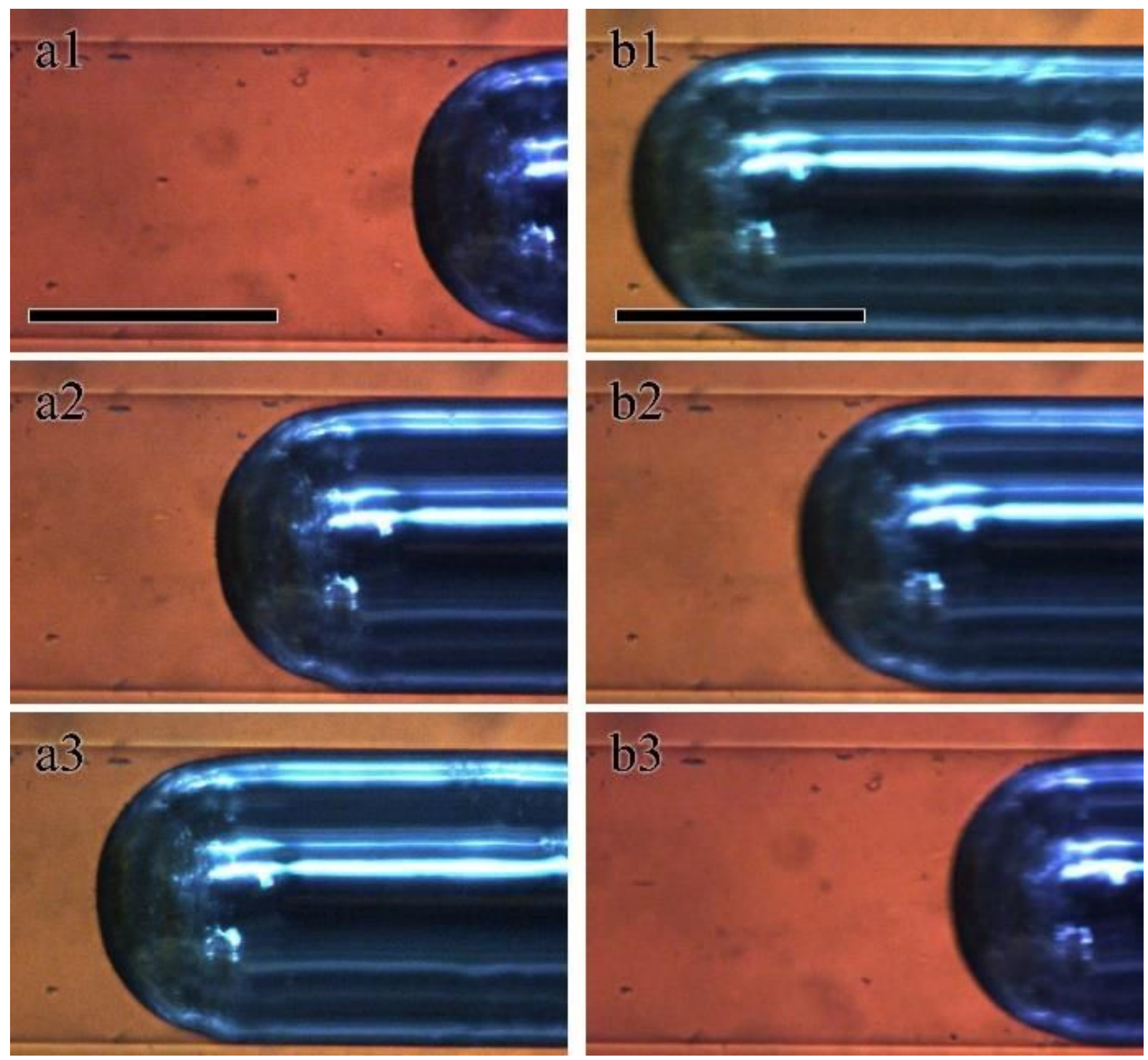

Figure 2

(a1-3) Optical microscopy images of Ga-LMs in silicone oil under positive pressure. The snapshots were taken every $5 \mathrm{~s}$. (b1-b3) Optical microscopy images of Ga-LMs in silicone oil under negative pressure. Snapshots were taken every $1 \mathrm{~s}$. The scale bars indicate $500 \mu \mathrm{m}$. 


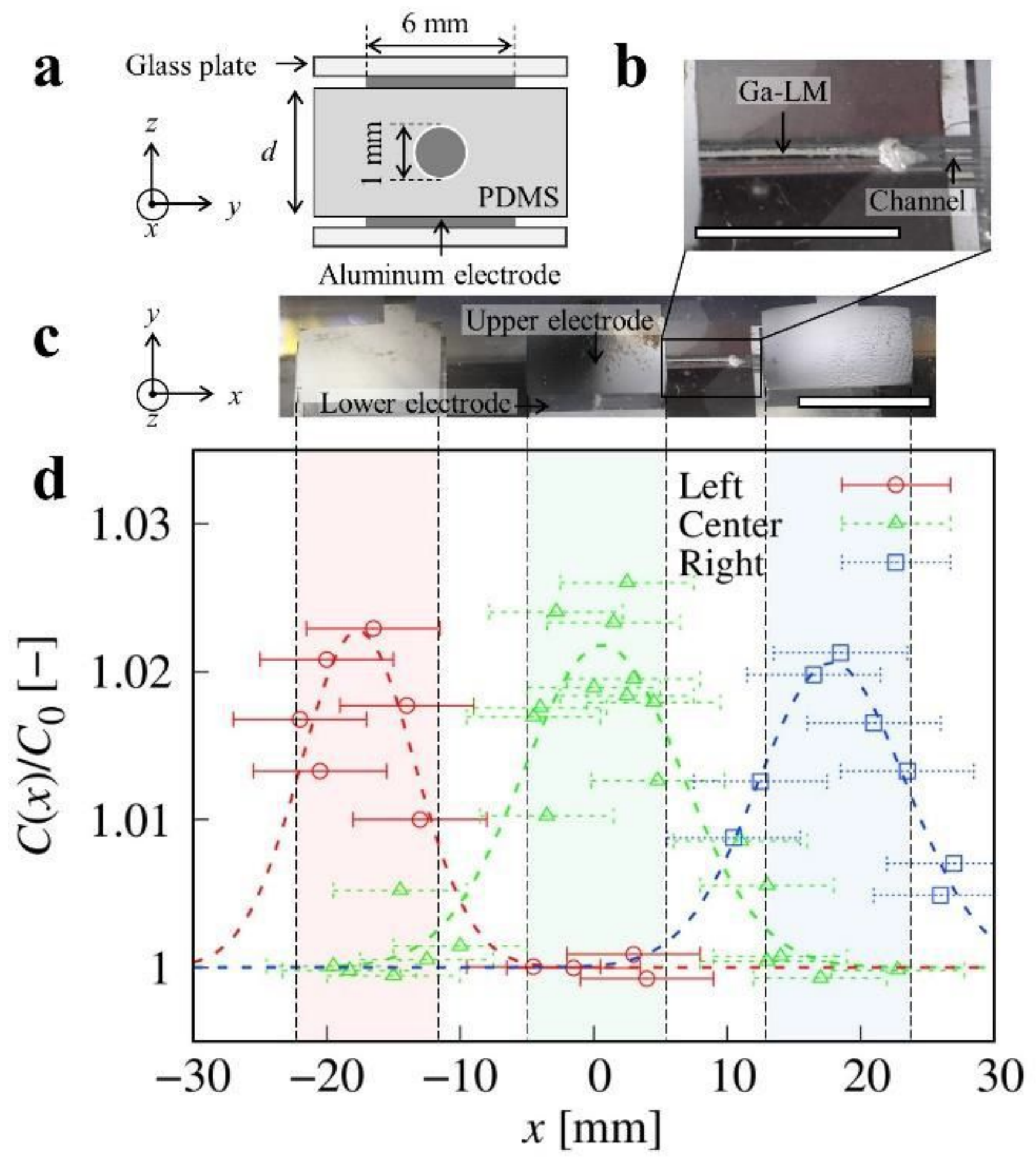

Figure 3

(a) Schematic cross section perpendicular to the microchannel. (b) An enlarged photograph of Ga-LMs in the microfluidic channel. The diameter of the channel is $1.0 \mathrm{~mm}$. The scale bar indicates $5 \mathrm{~mm}$. (c) A perspective photograph of the serially arranged capacitive device. The channel was sandwiched by upper and lower aluminum electrodes at three points, that is, at the left, center, and right. The scale bar indicates $10 \mathrm{~mm}$. (d) Normalized capacitance $\mathrm{C}(\mathrm{x}) / \mathrm{CO}$ as a function of the position of Ga-LMs, $\mathrm{x}$. Red, green, and 
blue markers indicate the capacitance measured at the left, center, and right electrodes, respectively. We provided a visual guide to indicate the position of the electrodes.

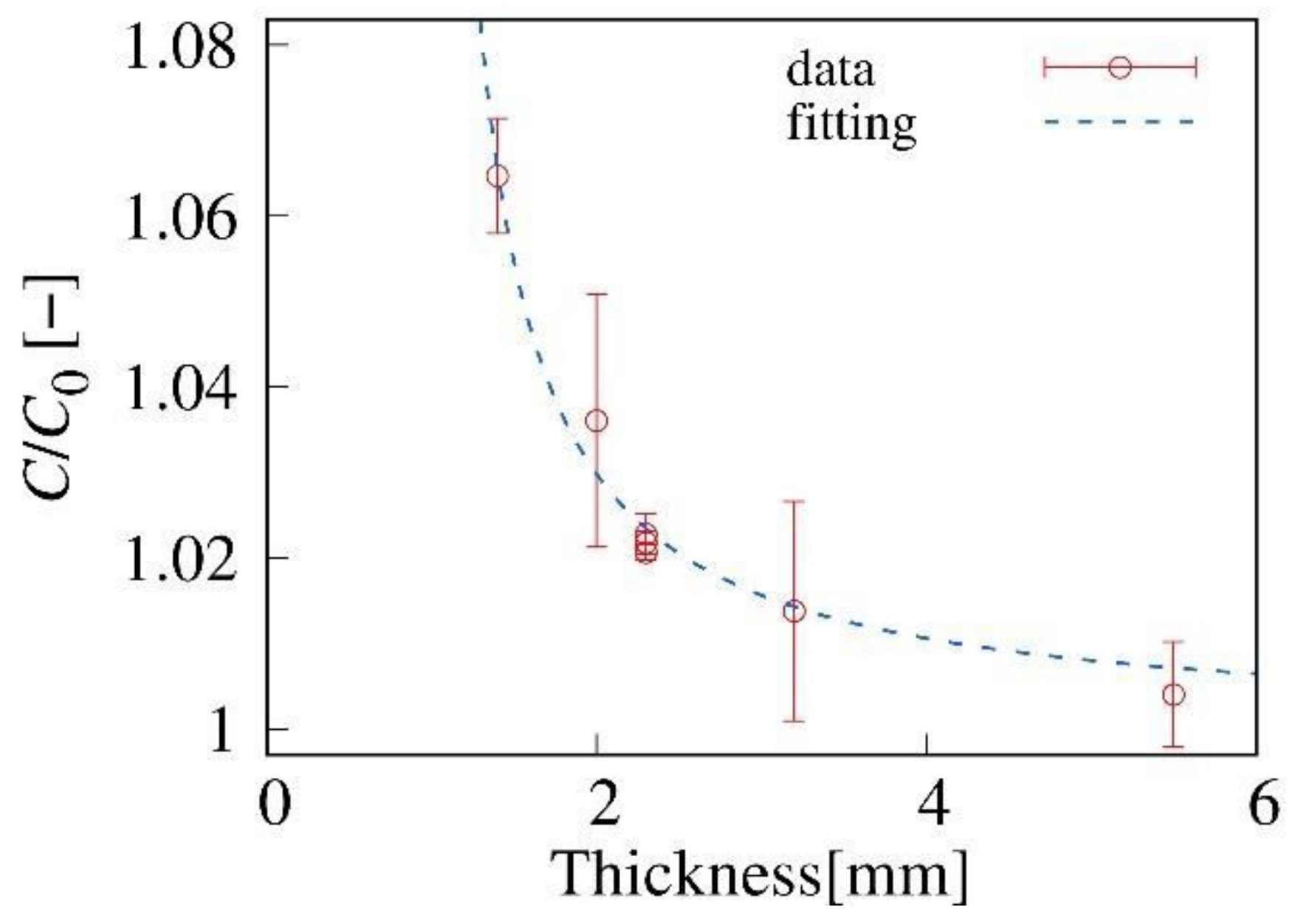

Figure 4

Dependence of the capacitance of Ga-LMs on the PDMS thickness. Red and blue markers indicate the experimental results measured by uniform electrodes and the maximum change estimated from the results shown in Fig. 3 (c), respectively. The diameter of the channel is $1 \mathrm{~mm}$. The green line is the best fit curve obtained using equation (5.a).

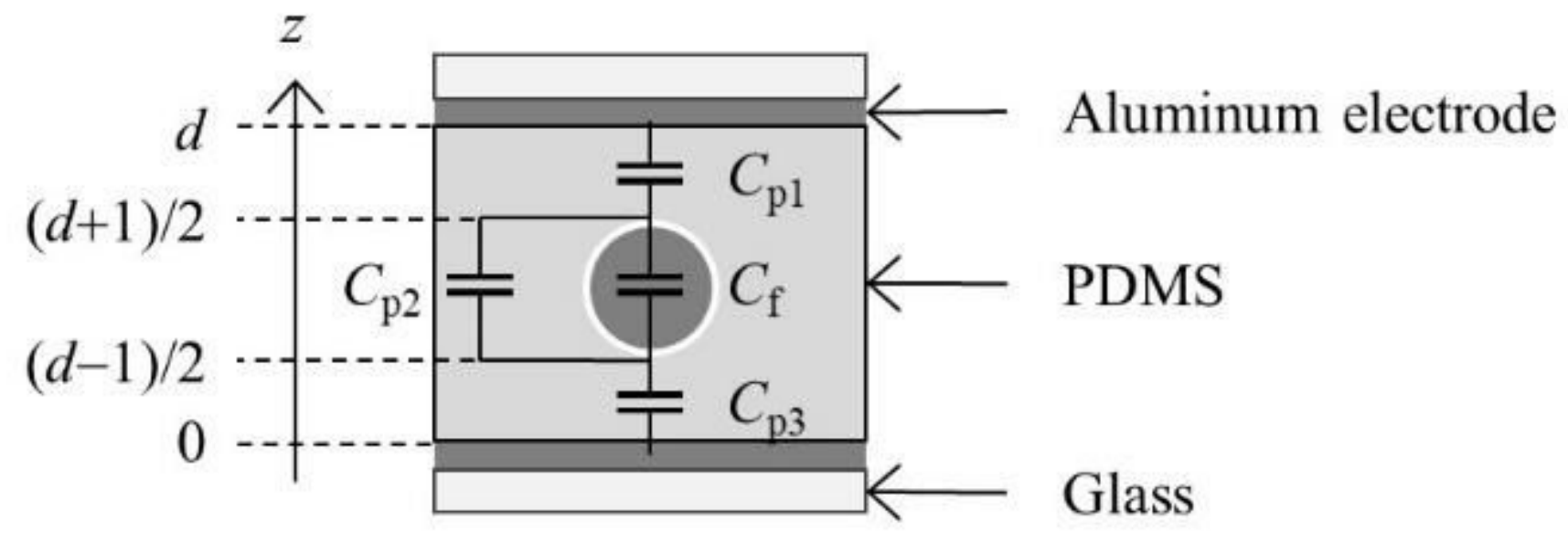


Figure 5

Schematic cross section of the channel and the equivalent circuit.

a1
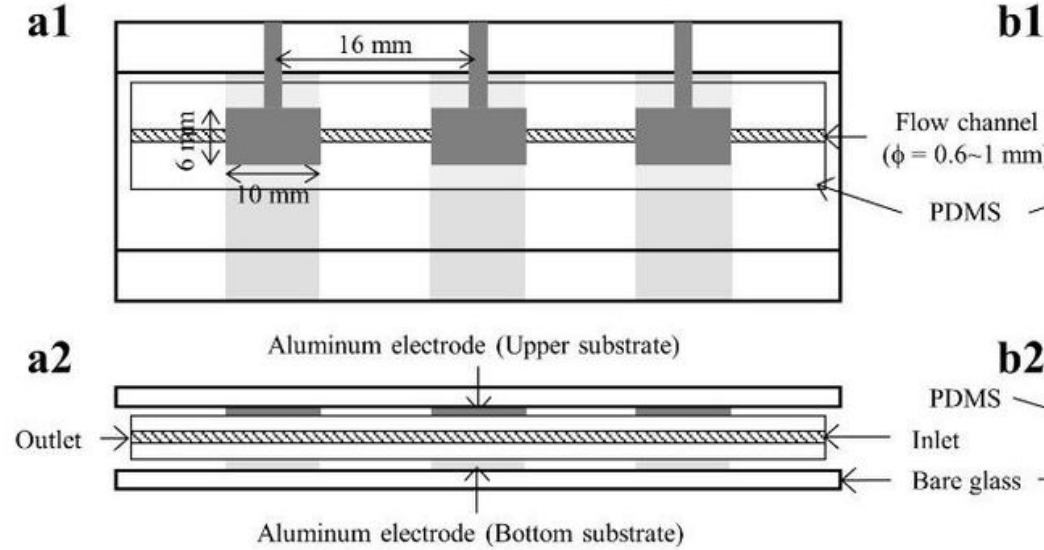

b1

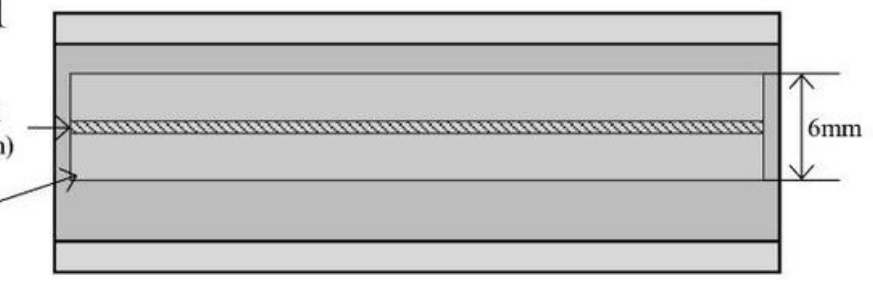

b2

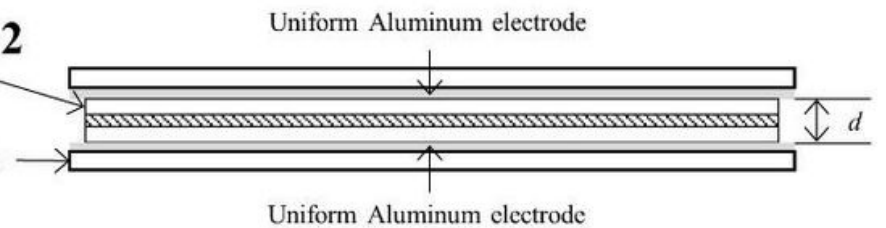

Figure 6

(a1) Schematic top view and (a2) schematic side view of the patterned electrode. We deposited three aluminum electrodes with dimensions of $6 \mathrm{~mm} * 10 \mathrm{~mm}$ on the upper glass substrate with intervals of 16 $\mathrm{mm}$. We deposited three aluminum electrodes whose width was $10 \mathrm{~mm}$ on the lower glass substrate with an interval of $16 \mathrm{~mm}$. (b1) Schematic top view and (b2) schematic side view of uniform electrodes. 